Amina Angelika Bouchentouf • Abdelhak Guendouzi

\title{
The $M^{X} / M / c$ Bernoulli feedback queue with variant multiple working vacations and impatient customers: performance and economic analysis
}

Received: 12 July 2018 / Accepted: 6 June 2019 / Published online: 18 June 2019

(C) The Author(s) 2019

\begin{abstract}
The present paper deals with an $M^{X} / M / c$ Bernoulli feedback queueing system with variant multiple working vacations and impatience timers which depend on the states of the servers. Whenever a customer arrives at the system, he activates an random impatience timer. If his service has not been completed before his impatience timer expires, the customer may abandon the system. Using certain customer retention mechanism, the impatient customer can be retained in the system. After getting incomplete or unsatisfactory service, with some probability, each customer may comeback to the system as a Bernoulli feedback. Using the probability generating functions (PGFs), we derive the steady-state solution of the model. Then, we obtain useful performance measures. Moreover, we carry out an economic analysis. Finally, numerical study is performed to explore the effects of the model parameters on the behavior of the system.
\end{abstract}

Mathematics Subject Classification $\quad 60 \mathrm{~K} 25 \cdot 68 \mathrm{M} 20 \cdot 90 \mathrm{~B} 22$

\section{Introduction}

Vacation queueing models with impatient customers are very helpful in providing basic framework for efficient design and study of diverse practical situations including telephone switchboard, inventory problems with perishable goods, computer and communication network, data/voice transmission, manufacturing system, etc.

In recent past, vacation queueing models have been widely studied. Doshi [3], Takagi [17], and Tian and Zhang [18] are excellent survey works on the subject. An extensive amount of the literature is available on queueing models with server vacation and batch arrival and can be found in Madan and AI-Rawwash [13], Wang et al. [20], Haridass and Arrumuganathan [5], etc.

Working vacation queues with customer impatience have attracted the interest of many researchers. Altman and Yechiali [1] treated the infinite-server queueing model with system's additional tasks and impatient customers. Perel and Yechiali [14] considered a two-phase Markovian random environment with impatient customers. Working vacation queueing model with customer impatience has been analyzed by Yue et al. [22]. Then, Zhang et al. [25] presented an equilibrium balking strategies in Markovian queues with working vacations. Sun et al. [16] gave the equilibrium and optimal behavior of customers in Markovian queues with multiple working vacations. Goswami [4] analyzed a queueing model with impatient customers with Bernoulli

A. A. Bouchentouf $(\varangle)$

Department of Mathematics, Mathematics Laboratory, Djillali Liabes University of Sidi Bel Abbes, B. P. 89,

22000 Sidi Bel Abbés, Algeria

E-mail: bouchentouf_amina@yahoo.fr

A. Guendouzi

Laboratory of Stochastic Models, Statistic and Applications, Dr Moulay Tahar University of Saida, En-Nasr, B. P. 138,

20000 Sidon, Algeria

E-mail: a.guendouzi@yahoo.com 
schedule working vacations and vacation interruption. Laxmi and Jyothsna [10] dealt with balking and reneging multiple working vacations queue with heterogeneous servers. Later, Tian et al. [19] presented equilibrium and optimal strategies in $M / M / 1$ queueing model with working vacations and vacation interruptions. Recently, in Bouchentouf and Yahiaoui [2], a study on queueing system with Bernoulli feedback, reneging and retention of reneged customers, multiple working vacations, and Bernoulli schedule vacation interruption has been done. For more literature on customer impatience in working vacation queueing models, the authors may be referred to Selvaraju and Goswami [15] and Laxmi and Jyothsna [8,9].

Variant of multiple vacation policy is relatively a recent one where it is permitted to the server to take a certain fixed number of consecutive vacations, if the system remains empty at the end of a vacation. This sort of vacation schedule was carried out by Zhang and Tian [24]. In their paper, a Geo/G/1 queueing model with multiple adaptive vacations has been analyzed. Literature related to variant multiple working vacations can be found in Ke [6], Ke et al. [7], Wang et al. [21], and Yue et al. [23]. Recently, Laxmi and Rajesh [11] studied a variant working vacations queue with customer impatience. Furthermore, the performance measures of batch arrival queue with variant working vacations and reneging have been presented in Laxmi and Rajesh [12].

In the present investigation, we carry out the analysis of an $M^{X} / M / c$ Bernoulli feedback queueing model with variant of multiple working vacations, reneging which depend on the states of the servers and retention of reneged customers. The queueing model presented in this paper has many practical applications. Moreover, as the impatience has strongly bad effect on the economy of any firm, a great idea of retention of impatient customers is incorporated in this work. Besides, to the best of our knowledge, modeling of multi-server queueing system with Bernoulli feedback, variant of working vacations, impatience timers which depend on the states of the servers, and retention of reneged customers has not been attempted in the literature. This paper makes a contribution in this sense.

The paper is arranged as follows. We describe the model in Sect. 2. The theoretical analysis of the system is presented in Sect. 3. Useful measures of effectiveness and the cost of our model are given in Sect. 4. To validate the analytical results and to facilitate the sensitivity analysis, we present some numerical results for system performance measures and cost model in Sect. 5. Some concluding remarks and notable features of investigation done are highlighted in Sect. 6.

\section{The model formulation}

We consider an $M^{X} / M / c$ queueing system with variant of working vacations, Bernoulli feedback, impatient customers which depend on the states of the servers, and retention of reneged customers. For the mathematical formulation of the queueing model, the following notations and assumptions are given:

Customers arrive in batches according to a Poisson process with rate $\lambda$. The arrival batch size $X$ is a random variable with probability mass function $P(X=l)=b_{l} ; l=1,2, \ldots$ The service times during normal busy period is assumed to be exponentially distributed with mean $1 / \mu$. During the vacation time, the service is provided according to an exponential distribution with parameter $\eta$, such that $\eta \leq \mu$. The queueing system consists of $c$ servers, all the servers go on working vacation synchronously once the system becomes empty, and they also return to the system as one at the same time. If the servers return from working vacation period to find an empty queue, they immediately leave all together for another working vacation. Otherwise, they return to serve the queue. Working vacation periods are assumed to be exponentially distributed with mean $1 / \phi$.

At a working vacation completion instant, if there are customers in the system, the servers come back to regular busy period. Otherwise, they take all together working vacations sequentially until the maximum number of working vacations, denoted by $K$ is taken. When the $K$ consecutive working vacations are complete, all servers switch to normal busy period and stay idle or busy depending on the availability of the arriving batches of customers. Therefore, in variant multiple vacation policy, if the system remains empty at the end of a working vacation, the servers are permitted to take a finite number, say $K$, of consecutive working vacations.

Whenever a batch of customers arrives to the system and finds the servers on working vacation (respectively. busy), an independent impatience timer $T_{1}$ (respectively. $T_{2}$ ) is activated, which is exponentially distributed with parameter $\xi_{1}$ (respectively. $\xi_{2}$ ). If the customer's service has not been completed before the customer's timer expires, the customer may leave the system. Each impatient customer may abandon the system with probability $\alpha$, and can be retained in the queue with complementary probability $\alpha^{\prime}=(1-\alpha)$. If the service is incomplete or unsatisfactory, the customer can either abandon the system with probability $\beta$ or rejoin the tail of the queue of the system for another service with probability $\beta^{\prime}$, where $\beta+\beta^{\prime}=1$. Note that, both customers, the newly arrived and those that are fed back are served in order in which they join the tail of the primary queue. 
The inter-arrival times, batch sizes, working vacation times, service times, and impatience times are independent of each other.

\section{Theoretical analysis of the model}

Let $N(t)$ denote the number of customers in the system at time $t$, and let $\kappa(t)$ be the status of the servers at time $t$. For the mathematical representation of the proposed model at an instant $t$, we consider the following states of the system based on the status of the servers:

$$
\kappa(t)=\left\{\begin{array}{l}
j, \text { the servers are taking the }(j+1) \text { th vacation at time } t, j=\overline{0,1, K-1}, \\
K, \text { the servers are idle or busy at time } t .
\end{array}\right.
$$

Figure 1 depicts the state-transition diagram. The bi-variate process $\{(N(t), \kappa(t)), t \geq 0\}$ represents two-dimensional infinite state Markov chain in continuous time with state space:

$$
\Omega=\{(n, j): n \geq 0 ; j=\overline{0, K}\}
$$

Let $P_{n, j}=\lim _{t \rightarrow \infty} P(N(t)=n ; \kappa(t)=j), n \geq 0 ; j=\overline{0, K}$ be the steady-state probabilities of the process $\{(N(t) ; \kappa(t)) ; t \geq 0\}$.

\subsection{PGFs and balance equations}

Define the probability generating functions as follows:

$$
\begin{aligned}
& G_{j}(z)=\sum_{n=0}^{\infty} P_{n, j} z^{n},|z| \leq 1, j=0, \ldots, K, \\
& G_{j}^{\prime}(z)=\frac{\mathrm{d}}{\mathrm{d} z} G_{j}(z)=\sum_{n=1}^{\infty} n P_{n, j} z^{n-1}, j=0, \ldots, K,
\end{aligned}
$$

and

$$
B(z)=\sum_{n=1}^{\infty} b_{n} z^{n}, \quad \text { with } \quad B(1)=\sum_{n=1}^{\infty} b_{n}=1 .
$$

To develop the model, the steady-state Chapman-Kolmogorov equations for the system states are constructed as follows:

$$
\begin{aligned}
(\lambda+\phi) P_{0,0} & =\left(\beta v+\alpha \xi_{1}\right) P_{1,0}+\left(\beta \mu+\alpha \xi_{2}\right) P_{1, K}, \\
\left(\lambda+\phi+\beta v+\alpha \xi_{1}\right) P_{1,0} & =\lambda b_{1} P_{0,0}+2\left(\beta v+\alpha \xi_{1}\right) P_{2,0}, \\
\left(\lambda+\phi+n\left(\beta v+\alpha \xi_{1}\right)\right) P_{n, 0}= & \lambda \sum_{m=1}^{n} b_{m} P_{n-m, 0}+(n+1)\left(\beta v+\alpha \xi_{1}\right) P_{n+1,0}, \\
& \quad 2 \leq n \leq c-1, \\
\left(\lambda+\phi+c \beta v+n \alpha \xi_{1}\right) P_{n, 0}= & \lambda \sum_{m=1}^{n} b_{m} P_{n-m, 0}+\left(c \beta v+(n+1) \alpha \xi_{1}\right) P_{n+1,0}, \quad n \geq c, \\
(\lambda+\phi) P_{0, j}= & \left(\beta v+\alpha \xi_{1}\right) P_{1, j}+\phi P_{0, j-1}, 1 \leq j \leq K, \\
\left(\lambda+\phi+\beta v+\alpha \xi_{1}\right) P_{1, j}= & \lambda b_{1} P_{0, j}+2\left(\beta v+\alpha \xi_{1}\right) P_{2, j}, 1 \leq j \leq K-1, \\
\left(\lambda+\phi+n\left(\beta v+\alpha \xi_{1}\right)\right) P_{n, j}= & \lambda \sum_{m=1}^{n} b_{m} P_{n-m, j}+(n+1)\left(\beta v+\alpha \xi_{1}\right) P_{n+1, j}, \\
& 2 \leq n \leq c-1,1 \leq j \leq K-1,
\end{aligned}
$$




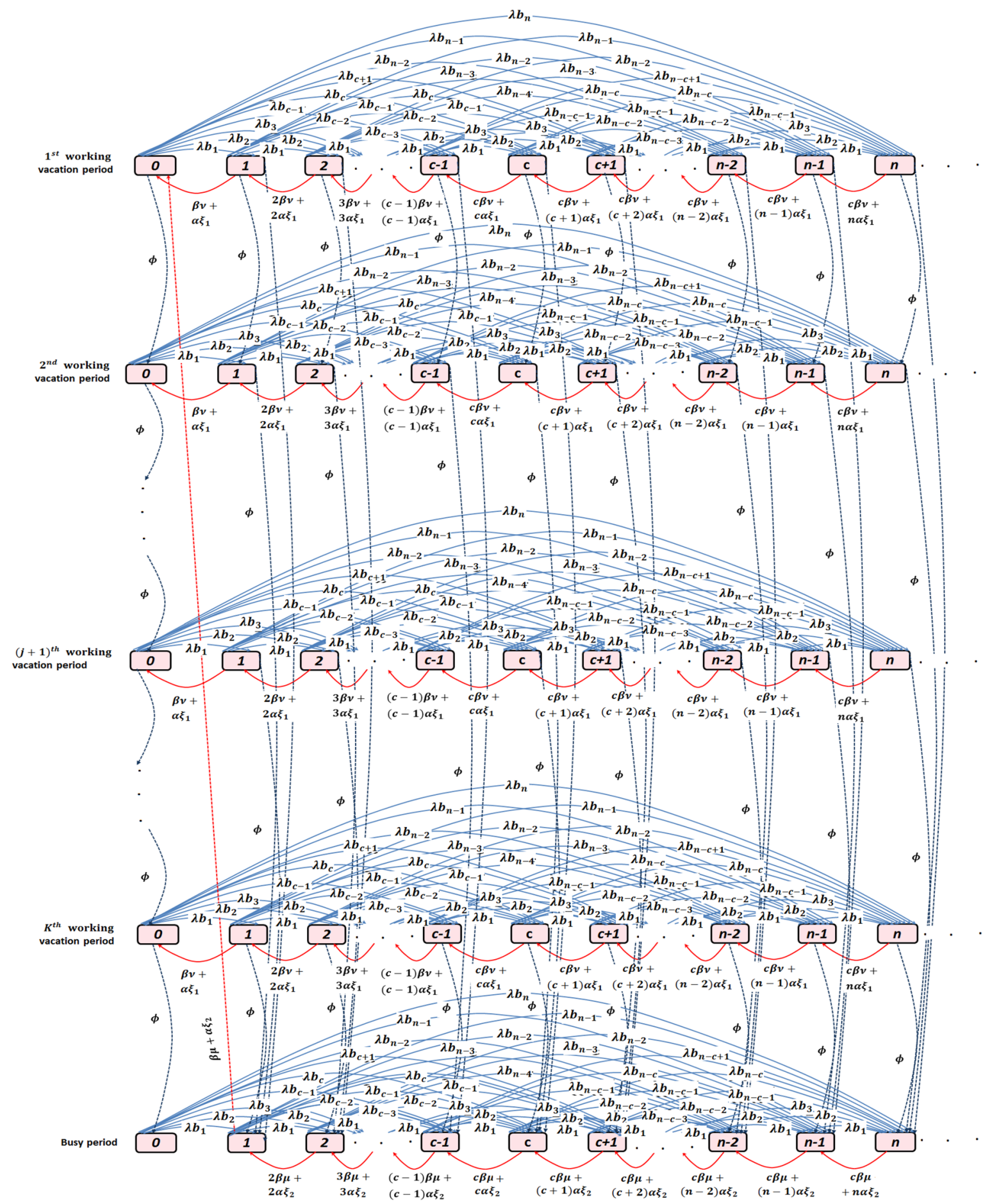

Fig. 1 State-transition-rate diagram

$$
\begin{gathered}
\left(\lambda+\phi+c \beta v+n \alpha \xi_{1}\right) P_{n, j}=\lambda \sum_{m=1}^{n} b_{m} P_{n-m, j}+\left(c \beta v+(n+1) \alpha \xi_{1}\right) P_{n+1, j}, \\
n \geq c, 1 \leq j \leq K-1, \\
\lambda P_{0, K}=\phi P_{0, K-1},
\end{gathered}
$$




$$
\begin{gathered}
\left(\lambda+\beta \mu+\alpha \xi_{2}\right) P_{1, K}=\lambda b_{1} P_{0, K}+2\left(\beta \mu+\alpha \xi_{2}\right) P_{2, K}+\phi \sum_{j=0}^{K-1} P_{1, j}, \\
\left(\lambda+n\left(\beta \mu+\alpha \xi_{2}\right)\right) P_{n, K}=\lambda \sum_{m=1}^{n} b_{m} P_{n-m, K}+(n+1)\left(\beta \mu+\alpha \xi_{2}\right) P_{n+1, K} \\
+\phi \sum_{j=0}^{K-1} P_{n, j}, 2 \leq n \leq c-1, \\
\left(\lambda+c \beta \mu+n \alpha \xi_{2}\right) P_{n, K}=\lambda \sum_{m=1}^{n} b_{m} P_{n-m, K}+\left(c \beta \mu+(n+1) \alpha \xi_{2}\right) P_{n+1, K} \\
+\phi \sum_{j=0}^{K-1} P_{n, j}, n \geq c .
\end{gathered}
$$

The normalizing condition is given as follows:

$$
\sum_{n=0}^{\infty} \sum_{j=0}^{K} P_{n, j}=1 .
$$

Multiplying Eqs. (1)-(4) by $z^{n}$ and summing all possible values of $n$, we get the following:

$$
\begin{gathered}
(1-z) z \alpha \xi_{1} G_{0}^{\prime}(z)+[\lambda z(B(z)-1)-z(\phi+c \beta v)+c \beta \nu] G_{0}(z) \\
=\beta v(1-z) \sum_{n=0}^{c-1}(c-n) P_{n, 0} z^{n}-\left(\alpha \xi_{2}+\beta \mu\right) z P_{1, K} .
\end{gathered}
$$

In a similar manner, we get from Eqs. (5)-(8):

$$
\begin{aligned}
& (1-z) z \alpha \xi_{1} G_{j}^{\prime}(z)+[\lambda z(B(z)-1)-z(\phi+c \beta v)+c \beta v] G_{j}(z) \\
& =\beta v(1-z) \sum_{n=0}^{c-1}(c-n) P_{n, j} z^{n}-\phi z P_{0, j-1}, \quad 1 \leq j \leq K-1 .
\end{aligned}
$$

In the same way, from Eqs. (9)-(12), we find the following:

$$
\begin{gathered}
(1-z) z \alpha \xi_{2} G_{K}^{\prime}(z)+[\lambda z(B(z)-1)+c \beta \mu(1-z)] G_{K}(z)=-z \phi \sum_{j=0}^{K-1} G_{j}(z) \\
\beta \mu(1-z) \sum_{n=0}^{c-1}(c-n) P_{n, K} z^{n}+z\left(\beta \mu+\alpha \xi_{2}\right) P_{1, K}+z \phi \sum_{j=0}^{K-2} P_{0, j} .
\end{gathered}
$$

Next, using the recursive method, we get the following:

$$
\left\{\begin{array}{l}
P_{n, 0}=\gamma_{n} P_{0,0}+\varphi_{n} P_{1, K}, \\
P_{n, j}=\gamma_{n} P_{0, j}+\omega_{n} P_{0, j-1},
\end{array}\right.
$$

where

$$
\gamma_{n}= \begin{cases}1, & \text { if } n=0 \\ \frac{\lambda+\phi}{\beta v+\alpha \xi 1}, & \text { if } n=1 . \\ \psi_{n-1} \gamma_{n-1}-\frac{A}{n} \sum_{i=1}^{n-1} b_{i} \gamma_{n-1-i} & \text { if } 2 \leq n \leq c-1,\end{cases}
$$




$$
\begin{gathered}
\varphi_{n}= \begin{cases}0, & \text { if } n=0 ; \\
-\frac{\beta \mu+\alpha \xi_{2}}{\beta v+\alpha \xi_{1}}, & \text { if } n=1 . \\
\psi_{n-1} \varphi_{n-1}-\frac{A}{n} \sum_{i=1}^{n-1} b_{i} \varphi_{n-1-i} & \text { if } 2 \leq n \leq c-1,\end{cases} \\
\omega_{n}= \begin{cases}0, & \text { if } n=0 ; \\
-\frac{\phi}{\beta v+\alpha \xi_{1}}, & \text { if } n=1 . \\
\psi_{n-1} \omega_{n-1}-\frac{A}{n} \sum_{i=1}^{n-1} b_{i} \omega_{n-1-i} & \text { if } 2 \leq n \leq c-1,\end{cases}
\end{gathered}
$$

with

$$
A=\frac{\lambda}{\beta v+\alpha \xi_{1}} \text { and } \psi_{n}=\frac{\lambda+\phi+n\left(\beta v+\alpha \xi_{1}\right)}{(n+1)\left(\beta v+\alpha \xi_{1}\right)}
$$

3.2 Solutions of the differential equations

For $z \neq 1$ and $z \neq 0$, Eqs. (14) and (15) can be written, respectively, as follows:

$$
\begin{gathered}
G_{0}^{\prime}(z)+\left(\frac{\lambda}{\alpha \xi_{1}} H^{\prime}(z)-\frac{(\phi+c \beta v)}{(1-z) \alpha \xi_{1}}+\frac{c \beta v}{(1-z) z \alpha \xi_{1}}\right) G_{0}(z) \\
=\frac{\beta v}{z \alpha \xi_{1}} Q_{0}(z) P_{0,0}+\left(\frac{\beta v}{\alpha \xi_{1} z} Q_{1}(z)-\frac{\alpha \xi_{2}+\beta \mu}{(1-z) \alpha \xi_{1}}\right) P_{1, K},
\end{gathered}
$$

for $j=\overline{1, K-1}$.

$$
\begin{aligned}
& G_{j}^{\prime}(z)+\left(\frac{\lambda}{\alpha \xi_{1}} H^{\prime}(z)-\frac{(\phi+c \beta v)}{(1-z) \alpha \xi_{1}}+\frac{c \beta v}{(1-z) z \alpha \xi_{1}}\right) G_{j}(z) \\
& =\frac{\beta v}{z \alpha \xi_{1}} Q_{0}(z) P_{0, j}+\left(\frac{\beta v}{\alpha \xi_{1} z} Q_{2}(z)-\frac{\phi}{(1-z) \alpha \xi_{1}}\right) P_{0, j-1}
\end{aligned}
$$

where

$$
Q_{0}(z)=\sum_{n=0}^{c-1}(c-n) \gamma_{n} z^{n}, Q_{1}(z)=\sum_{n=0}^{c-1}(c-n) \varphi_{n} z^{n}, Q_{2}(z)=\sum_{n=0}^{c-1}(c-n) \omega_{n} z^{n}
$$

with

$$
H(z)=\int_{0}^{z} \frac{B(x)-1}{1-x} \mathrm{~d} x \text { and } H^{\prime}(z)=\frac{B(z)-1}{1-z} .
$$

Now, by taking $z=1$ in Eqs. (14) and (15), we, respectively, have the following:

$$
\phi G_{0}(1)=\left(\alpha \xi_{2}+\beta \mu\right) P_{1, K},
$$

and

$$
G_{j}(1)=P_{0, j-1} .
$$

Next, to solve the linear differential Eqs. (17) and (18), we multiply both sides of the above equations by $e^{\frac{\lambda}{\alpha \xi_{1}} H(z)}(1-z)^{\frac{\phi}{\alpha \xi_{1}}} z^{\frac{c \beta \nu}{\alpha \xi_{1}}}$. Then, integrating form 0 to $z$, we obtain the following:

$$
G_{0}(z)=\frac{e^{-\frac{\lambda}{\alpha \xi_{1}} H(z)}}{(1-z)^{\frac{\phi}{\alpha \xi_{1}}} z^{\frac{c \beta v}{\alpha \xi_{1}}}}\left\{\frac{\beta v}{\alpha \xi_{1}} K_{0}(z) P_{0,0}+\left(\frac{\beta v}{\alpha \xi_{1}} K_{1}(z)-\frac{\alpha \xi_{2}+\beta \mu}{\alpha \xi_{1}} K_{2}(z)\right) P_{1, K}\right\}
$$


for $j=\overline{1, K-1}$.

$$
G_{j}(z)=\frac{e^{-\frac{\lambda}{\alpha \xi_{1}} H(z)}}{(1-z)^{\frac{\phi}{\alpha \xi_{1}}} z^{\frac{c \beta \nu}{\alpha \xi_{1}}}}\left\{\frac{\beta v}{\alpha \xi_{1}} K_{0}(z) P_{0, j}+\left(\frac{\beta v}{\alpha \xi_{1}} K_{3}(z)-\frac{\phi}{\alpha \xi_{1}} K_{2}(z)\right) P_{0, j-1}\right\},
$$

where

$$
\begin{aligned}
& K_{0}(z)=\int_{0}^{z} e^{\frac{\lambda}{\alpha \xi_{1}} H(x)}(1-x)^{\frac{\phi}{\alpha \xi_{1}}} x^{\frac{c \beta v}{\alpha \xi_{1}}-1} Q_{0}(x) \mathrm{d} x, \\
& K_{1}(z)=\int_{0}^{z} e^{\frac{\lambda}{\alpha \xi_{1}} H(x)}(1-x)^{\frac{\phi}{\alpha \xi_{1}}} x^{\frac{c \beta \nu}{\alpha \xi_{1}}-1} Q_{1}(x) \mathrm{d} x, \\
& K_{2}(z)=\int_{0}^{z} e^{\frac{\lambda}{\alpha \xi_{1}} H(x)}(1-x)^{\frac{\phi}{\alpha \xi_{1}}-1} x^{\frac{c \beta v}{\alpha \xi_{1}}} \mathrm{~d} x,
\end{aligned}
$$

and

$$
K_{3}(z)=\int_{0}^{z} e^{\frac{\lambda}{\alpha \xi_{1}} H(x)}(1-x)^{\frac{\phi}{\alpha \xi_{1}}} x^{\frac{c \beta \nu}{\alpha \xi_{1}}-1} Q_{2}(x) \mathrm{d} x .
$$

Next, $z=0$ and $z=1$ are the roots of the numerator of the right-hand sides of (21) and (22). Thus, taking $z=1$ in (21) and (22), respectively, we get the following:

$$
P_{1, K}=\theta_{1} P_{0,0}, \quad \text { where } \quad \theta_{1}=\frac{\beta \nu K_{0}(1)}{\left(\beta \mu+\alpha \xi_{2}\right) K_{2}(1)-\beta \nu K_{1}(1)},
$$

and

$$
P_{0, j}=C^{j} P_{0,0}, \quad 1 \leq j \leq K-1, \quad \text { where } C=\frac{\phi K_{2}(1)-\beta \nu K_{3}(1)}{\beta \nu K_{0}(1)} .
$$

Via Eqs. (9) and (24), we obtain the following:

$$
P_{0, K}=\theta_{0} P_{0,0}
$$

where

$$
\theta_{0}=\frac{\phi}{\lambda} C^{K-1}
$$

Substituting Eqs. (23) and (24) in Eqs. (21) and (22), respectively, we get the following:

$$
G_{0}(z)=\frac{e^{-\frac{\lambda}{\alpha \xi_{1}} H(z)}}{(1-z)^{\frac{\phi}{\alpha \xi_{1}}} z^{\frac{c \beta \nu}{\alpha \xi_{1}}}}\left\{\frac{\beta \nu K_{0}(z)+\left(\beta \nu K_{1}(z)-\left(\beta \mu+\alpha \xi_{2}\right) K_{2}(z)\right) \theta_{1}}{\alpha \xi_{1}}\right\} P_{0,0},
$$

and for $j=\overline{1, K-1}$

$$
G_{j}(z)=\frac{e^{-\frac{\lambda}{\alpha \xi_{1}} H} H(z)}{(1-z)^{\frac{\phi}{\alpha \xi_{1}}} z^{\frac{c \beta v}{\alpha \xi_{1}}}}\left\{\beta v K_{0}(z)+\frac{\beta v K_{3}(z)-\phi K_{2}(z)}{C}\right\} \frac{C^{j}}{\alpha \xi_{1}} P_{0,0} .
$$

Thus

$$
\sum_{j=0}^{K-1} G_{j}(z)=\Psi(z) P_{0,0}, \quad j=\overline{0, K-1}
$$

with

$$
\begin{aligned}
\Psi(z)= & \frac{e^{-\frac{\lambda}{\alpha \xi_{1}} H(z)}}{(1-z)^{\frac{\phi}{\alpha \xi_{1}}} z^{\frac{c \beta \nu}{\alpha \xi_{1}}}}\left\{\frac{\beta \nu K_{0}(z)+\left(\beta \nu K_{1}(z)-\left(\beta \mu+\alpha \xi_{2}\right) K_{2}(z)\right) \theta_{1}}{\alpha \xi_{1}}\right. \\
& \left.+\frac{C}{\alpha \xi_{1}}\left(\beta \nu K_{0}(z)+\frac{\beta \nu K_{3}(z)-\phi K_{2}(z)}{C}\right)\left(\frac{1-C^{K-1}}{1-C}\right)\right\} .
\end{aligned}
$$


By taking $z=1$ in Eq. (16), we find the following:

$$
\phi \sum_{j=0}^{K-1} G_{j}(1)=\left(\beta \mu+\alpha \xi_{2}\right) P_{1, K}+\phi \sum_{j=0}^{K-2} P_{0, j} .
$$

Consequently, we have the following:

$$
\sum_{j=0}^{K-1} G_{j}(1)=\left\{\frac{\beta \mu+\alpha \xi_{2}}{\phi} \theta_{1}+\frac{1-C^{K-1}}{1-C}\right\} P_{0,0} .
$$

Next, we have to solve the differential Eq. (16). Therefore, we must express recursively the quantity $P_{n, K}$ in terms of $P_{0,0}$. In the same manner as previously, it yields the following:

$$
P_{n, K}=\theta_{n} P_{0,0},
$$

with

$$
\theta_{n}= \begin{cases}\theta_{0}, & \text { if } n=0 ; \\ \theta_{1}, & \text { if } n=1 . \\ \sigma_{n-1} \theta_{n-1}-\frac{B}{n} \sum_{i=1}^{n-1} b_{i} \theta_{n-1-i}-\frac{E}{n}\left(\gamma_{n-1} H(K)+\omega_{n-1} h(K)\right) & \text { if } 2 \leq n \leq c-1,\end{cases}
$$

where

$$
\begin{gathered}
\sigma_{n}=\frac{\lambda+n\left(\beta \mu+\alpha \xi_{2}\right)}{(n+1)\left(\beta \mu+\alpha \xi_{2}\right)}, \quad B=\frac{\lambda}{\beta \mu+\alpha \xi_{2}}, \quad E=\frac{\phi}{\beta \mu+\alpha \xi_{2}}, \\
H(K)=\sum_{j=0}^{K-1} C^{j}=\frac{1-C^{K}}{1-C}, \quad \text { and } h(K)=\sum_{j=0}^{K-1} C^{j-1}=\frac{1-C^{K}}{C(1-C)} .
\end{gathered}
$$

By substituting Eqs. (29) in (16), we have the following:

$$
G_{K}^{\prime}(z)+\left(\frac{\lambda}{\alpha \xi_{2}} H(z)^{\prime}+\frac{c \beta \mu}{z \alpha \xi_{2}}\right) G_{K}(z)=\frac{\beta \mu}{z \alpha \xi_{2}} Q_{3}(z) P_{0,0}-\frac{\phi \sum_{j=0}^{K-1}\left[G_{j}(z)-G_{j}(1)\right]}{(1-z) \alpha \xi_{2}},
$$

where

$$
Q_{3}(z)=\sum_{n=0}^{c-1}(c-n) \theta_{n} z^{n} .
$$

Multiplying Eq. (31) by $e^{\frac{\lambda}{\alpha \xi_{2}}} H(z) \frac{c \beta \mu}{\frac{\alpha \beta}{\alpha \xi_{2}}}$ and integrating from 0 to $z$, then using Eqs. (28) and (30), we get the following:

$$
G_{K}(z)=\frac{e^{-\frac{\lambda}{\alpha \xi_{2}} H(z)}}{z^{\frac{c \beta \mu}{\alpha \xi_{2}}}}\left\{-\frac{\phi}{\alpha \xi_{2}}\left(K_{4}(z)-\left(\frac{\beta \mu+\alpha \xi_{2}}{\phi} \theta_{1}+\frac{1-C^{K-1}}{1-C}\right) K_{5}(z)\right)+\frac{\beta \mu}{\alpha \xi_{2}} K_{6}(z)\right\} P_{0,0},
$$

where

$$
\begin{aligned}
& K_{4}(z)=\int_{0}^{z} e^{\frac{\lambda}{\alpha \xi_{2}} H(x)} x^{\frac{c \beta \mu}{\alpha \xi_{2}}}(1-x)^{-1} \Psi(x) \mathrm{d} x, \\
& K_{5}(z)=\int_{0}^{z} e^{\frac{\lambda}{\alpha \xi_{2}} H(x)} x^{\frac{c \beta \mu}{\alpha \xi_{2}}}(1-x)^{-1} \mathrm{~d} x,
\end{aligned}
$$

and

$$
K_{6}(z)=\int_{0}^{z} e^{\frac{\lambda}{\alpha \xi_{2}} H(x)} x^{\frac{c \beta \mu}{\alpha \xi_{2}}-1} Q_{3}(x) \mathrm{d} x .
$$


Now, taking $z=1$ in Eq. (32), and using the normalization condition:

$$
\sum_{j=0}^{K-1} G_{j}(1)+G_{K}(1)=1,
$$

we obtain the following:

$$
\begin{aligned}
P_{0,0}= & \left\{e ^ { - \frac { \lambda } { \alpha \xi _ { 2 } } H ( 1 ) } \left\{-\frac{\phi}{\alpha \xi_{2}}\left(K_{4}(1)-\left(\frac{\beta \mu+\alpha \xi_{2}}{\phi} \theta_{1}+\frac{1-C^{K-1}}{1-C}\right) K_{5}(1)\right)\right.\right. \\
& \left.\left.+\frac{\beta \mu}{\alpha \xi_{2}} K_{6}(1)\right\}+\left(\frac{\beta \mu+\alpha \xi_{2}}{\phi} \theta_{1}+\frac{1-C^{K-1}}{1-C}\right)\right\}^{-1} .
\end{aligned}
$$

\section{Performance measures and cost model}

\subsection{Measures of effectiveness}

Performance measures are significant features of queueing systems as they reflect the effectiveness of the considered queueing system. The queueing model developed may be of great importance using some useful characteristics which can be in the future employed for the prediction, development, and improvement of the concerned real-world queueing system. In this section, we formulate some important system performance measures in terms of steady-state probabilities.

- The average number of customers in the system $(E(L))$.

$$
E(L)=E\left(L_{\mathrm{WV}}\right)+E\left(L_{\mathrm{K}}\right),
$$

where $E\left(L_{\mathrm{WV}}\right)$ is the mean system size when the servers are on working vacation and $E\left(L_{\mathrm{K}}\right)$ represents the mean system size when the servers are in busy period. Differentiating Eq. (14), taking $z=1$, and using Eq. (19), we get the following:

$$
\left(\alpha \xi_{1}+\phi\right) G_{0}^{\prime}(1)=\left(\lambda B^{\prime}(1)-c \beta v\right) G_{0}(1)+\beta v\left(Q_{0}(1) P_{0,0}+Q_{1}(1) P_{1, K}\right) .
$$

In the same manner, for $j=\overline{1, K-1}$, differentiating Eq. (15), taking $z=1$ and using Eq. (20), we get the following:

$$
\left(\alpha \xi_{1}+\phi\right) G_{j}^{\prime}(1)=\left(\lambda B^{\prime}(1)-c \beta v\right) G_{j}(1)+\beta v\left(Q_{0}(1) P_{0, j}-Q_{2}(1) P_{0, j-1}\right),
$$

where

$$
Q_{0}(1)=\sum_{n=0}^{c-1}(c-n) \gamma_{n}, Q_{1}(1)=\sum_{n=0}^{c-1}(c-n) \varphi_{n}, Q_{2}(1)=\sum_{n=0}^{c-1}(c-n) \omega_{n} .
$$

From Eq. (34), we obtain the following:

$$
G_{0}^{\prime}(1)=\left\{\frac{\lambda B^{\prime}(1)-c \beta v}{\alpha \xi_{1}+\phi}\left(\frac{\beta \mu+\alpha \xi_{2}}{\phi}\right) \theta_{1}+\frac{\beta v\left(Q_{0}(1)+\theta_{1} Q_{1}(1)\right)}{\alpha \xi_{1}+\phi}\right\} P_{0,0} .
$$

From Eq. (35), summing over all the possible values of $j, j=\overline{1, K-1}$, we obtain the following:

$$
\sum_{j=1}^{K-1} G_{j}^{\prime}(1)=\left\{\left(\frac{1-C^{K-1}}{C(1-C)}\right) \frac{\lambda B^{\prime}(1)+\beta \nu\left(Q_{0}(1) C-Q_{2}(1)-c\right)}{\alpha \xi_{1}+\phi}\right\} P_{0,0} .
$$


Furthermore, $E\left(L_{\mathrm{WV}}\right)$ is obtained as follows:

$$
E\left(L_{\mathrm{WV}}\right)=G_{0}^{\prime}(1)+\sum_{j=1}^{K-1} G_{j}^{\prime}(1) .
$$

Substituting Eqs. (36) and (37) in (38), we get the following:

$$
\begin{aligned}
E\left(L_{\mathrm{WV}}\right)= & \left\{\frac{\lambda B^{\prime}(1)-c \beta v}{\alpha \xi_{1}+\phi}\left(\frac{\beta \mu+\alpha \xi_{2}}{\phi}\right) \theta_{1}+\frac{\beta v\left(Q_{0}(1)+\theta_{1} Q_{1}(1)\right)}{\alpha \xi_{1}+\phi}\right. \\
& \left.+\left(\left(\frac{1-C^{K-1}}{C(1-C)}\right) \frac{\lambda B^{\prime}(1)+\beta v\left(Q_{0}(1) C-Q_{2}(1)-c\right)}{\alpha \xi_{1}+\phi}\right)\right\} P_{0,0} .
\end{aligned}
$$

Next, from Eq. (16), using L'Hospital rule, we find the following:

$$
\begin{aligned}
E\left(L_{\mathrm{K}}\right)= & \lim _{z \rightarrow 1} G_{K}^{\prime}(z)=\lim _{z \rightarrow 1}\left\{\frac{-(\lambda z(B(z)-1)+c \beta \mu(1-z))}{(1-z) \alpha \xi_{2}} G_{K}(z)\right. \\
& \left.-\frac{\phi \sum_{j=0}^{K-1}\left[G_{j}(z)-G_{j}(1)\right]}{(1-z) \alpha \xi_{2}}+\frac{\beta \mu}{z \alpha \xi_{2}} Q_{3}(z) P_{0,0}\right\} .
\end{aligned}
$$

This implies that

$$
E\left(L_{\mathrm{K}}\right)=\frac{\lambda B^{\prime}(1)-\beta \mu}{\alpha \xi_{2}} G_{K}(1)+\frac{\phi}{\alpha \xi} \sum_{j=1}^{K-1} G_{j}^{\prime}(1)+\frac{\beta \mu}{\alpha \xi_{2}} Q_{3}(1) P_{0,0}
$$

where

$$
Q_{3}(1)=\sum_{n=0}^{c-1}(c-n) \theta_{n}
$$

- The mean number of customers in the queue $\left(E\left(L_{\mathrm{q}}\right)\right)$ :

$$
\begin{aligned}
E\left(L_{\mathrm{q}}\right) & =\sum_{j=0}^{K} \sum_{n=c+1}^{\infty}(n-c) P_{n, j} \\
& =E(L)-c+\left\{\left(Q_{0}(1)+\frac{Q_{2}(1)}{C}\right)\left(\frac{1-C^{K}}{1-C}\right)+Q_{3}(1)\right\} P_{0,0} .
\end{aligned}
$$

- The probability that the servers are in working vacation period $\left(P_{\mathrm{WV}}\right)$ :

$$
P_{\mathrm{WV}}=\sum_{j=0}^{K-1} G_{j}(1)=\left\{\frac{\beta \mu+\alpha \xi_{2}}{\phi} \theta_{1}+\frac{1-C^{K-1}}{1-C}\right\} P_{0,0} .
$$

- The probability that the servers are idle during working vacation period $\left(P_{\text {idle }}\right)$ :

$$
P_{\text {idle }}=\sum_{j=0}^{K-1} P_{0, j}=\frac{1-C^{K}}{1-C} P_{0,0} .
$$

- The probability that the servers are busy $\left(P_{\text {busy }}\right)$ :

$$
P_{\text {busy }}=1-P_{0, K}-P_{\mathrm{WV}} \text {. }
$$


Table 1 Total costs vs. $\lambda$

\begin{tabular}{|c|c|c|c|c|c|c|c|c|c|}
\hline \multirow{2}{*}{$\begin{array}{l}K \\
\lambda\end{array}$} & \multicolumn{3}{|l|}{1} & \multicolumn{3}{|l|}{5} & \multicolumn{3}{|l|}{9} \\
\hline & $\Gamma$ & $\Delta$ & $\Theta$ & $\Gamma$ & $\Delta$ & $\Theta$ & $\Gamma$ & $\Delta$ & $\Theta$ \\
\hline 2.9 & 120.4041 & 531.6658 & 411.2618 & 195.2688 & 568.5601 & 373.2913 & 195.3084 & 568.5796 & 373.2712 \\
\hline 3.0 & 122.1671 & 534.7137 & 412.5467 & 192.7104 & 569.8081 & 377.0976 & 192.7494 & 569.8274 & 377.0781 \\
\hline 3.1 & 123.9192 & 537.6298 & 413.7105 & 190.3974 & 571.0168 & 380.6194 & 190.4356 & 571.0360 & 380.6003 \\
\hline 3.2 & 125.6620 & 540.4197 & 414.7576 & 188.3150 & 572.1867 & 383.8717 & 188.3524 & 572.2056 & 383.8533 \\
\hline 3.3 & 126.7224 & 545.4875 & 418.7651 & 182.1972 & 573.0985 & 390.8506 & 182.2479 & 573.0985 & 390.5806 \\
\hline
\end{tabular}

- The mean number of customers served per unit time $\left(N_{\mathrm{s}}\right)$ :

$$
\begin{aligned}
N_{\mathrm{s}} & =\beta \mu \sum_{n=1}^{c-1} n P_{n, K}+c \beta \mu \sum_{n=c}^{\infty} P_{n, K}+\beta v \sum_{j=0}^{K} \sum_{n=1}^{c-1} n P_{n, j}+c \beta v \sum_{j=0}^{K} \sum_{n=c}^{\infty} P_{n, j} \\
& =c \beta\left(\mu\left(P_{\text {busy }}+P_{0, K}\right)+v P_{\mathrm{WV}}\right)+\beta\left(\mu Q_{3}(1)+v\left(Q_{0}(1) H(K)+Q_{2}(1) h(K)\right)\right) P_{0,0} .
\end{aligned}
$$

* The average rate of abandonment of a customer due to impatience $\left(R_{\mathrm{a}}\right)$ :

$$
\begin{aligned}
R_{\mathrm{a}} & =\sum_{j=0}^{K-1} \sum_{n=0}^{\infty} n \alpha \xi_{1} P_{n, j}+\sum_{n=0}^{\infty} n \alpha \xi_{2} P_{n, K} \\
& =\alpha \xi_{1} E\left(L_{\mathrm{WV}}\right)+\alpha \xi_{2} E\left(L_{\mathrm{K}}\right) .
\end{aligned}
$$

* The average rate of retention of impatient customers $\left(R_{\mathrm{e}}\right)$ :

$$
\begin{aligned}
R_{\mathrm{e}} & =\sum_{j=0}^{K-1} \sum_{n=0}^{\infty} n(1-\alpha) \xi_{1} P_{n, j}+\sum_{n=0}^{\infty} n(1-\alpha) \xi_{2} P_{n, K} \\
& =(1-\alpha) \xi_{1} E\left(L_{\mathrm{WV}}\right)+(1-\alpha) \xi_{2} E\left(L_{\mathrm{K}}\right) .
\end{aligned}
$$

\subsection{Economic model}

To construct the cost model, we consider the following cost (in unit) elements associated with different events:

- $C_{1}$ Cost per unit time when the servers are busy.

$-C_{2}$ Cost per unit time when the servers are idle during busy period.

- $C_{3}$ Cost per unit time when the servers are idle during working vacation period.

$-C_{4}$ Cost per unit time when the servers are on working vacation period.

- $C_{5}$ Cost per unit time when a customer joins the queue and waits for service.

- $C_{6}$ Cost per unit time when a customer reneges.

- $C_{7}$ Cost per unit time when a customer is retained.

- $C_{8}$ Cost per service per unit time when the servers are in busy period.

- $C_{9}$ Cost per service per unit time when the servers are in working vacation period.

- $C_{10}$ Cost per unit time when a customer returns to the system as a feedback customer.

- $C_{11}$ Fixed server purchase cost per unit.

Let $R$ be the revenue earned by providing service to a customer.

$\Gamma$ be the total expected cost per unit time of the system:

$$
\begin{aligned}
\Gamma= & C_{1} P_{\text {busy }}+C_{2} P_{0, K}+C_{3} P_{\text {idle }}+C_{4} P_{\mathrm{Wv}}+C_{5} E\left(L_{\mathrm{q}}\right)+C_{6} R_{\mathrm{a}}+C_{7} R_{\mathrm{e}} \\
& +c \mu C_{8}+c v C_{9}+c \beta^{\prime}(\mu+v) C_{10}+c C_{11} .
\end{aligned}
$$


Table 2 Total costs vs. $\phi$

\begin{tabular}{|c|c|c|c|c|c|c|c|c|c|}
\hline \multirow{2}{*}{$\begin{array}{l}q \\
\phi\end{array}$} & \multicolumn{3}{|l|}{0.5} & \multicolumn{3}{|l|}{0.7} & \multicolumn{3}{|l|}{0.9} \\
\hline & $\Gamma$ & $\Delta$ & $\Theta$ & $\Gamma$ & $\Delta$ & $\Theta$ & $\Gamma$ & $\Delta$ & $\Theta$ \\
\hline 0.07 & 160.2763 & 595.4973 & 435.2210 & 172.8560 & 572.8560 & 400.0426 & 216.3829 & 545.9906 & 329.6077 \\
\hline 0.09 & 160.7400 & 596.2907 & 435.5507 & 858 & 578.0479 & 407.7620 & 219.4602 & 554.4544 & 334.9942 \\
\hline 0.11 & 160.8474 & 596.8863 & 436.0389 & 166.8535 & 581.9042 & 415.0506 & 213.1461 & 565.2968 & 352.1507 \\
\hline 0.13 & 160.7960 & 597.3476 & 436.5516 & 161.7721 & 584.5145 & 422.7425 & 205.0141 & 571.0750 & 366.0609 \\
\hline 0.15 & 160.6748 & 597.7125 & 437.0377 & 158.9608 & 586.6848 & 427.7240 & 197.1398 & 575.5170 & 378.3772 \\
\hline
\end{tabular}

Table 3 Total costs vs. $\mu$

\begin{tabular}{|c|c|c|c|c|c|c|c|c|c|}
\hline \multirow{2}{*}{$\begin{array}{l}\xi_{2} \\
\mu\end{array}$} & \multicolumn{3}{|l|}{0.80} & \multicolumn{3}{|l|}{0.94} & \multicolumn{3}{|l|}{1.04} \\
\hline & $\Gamma$ & $\Delta$ & $\Theta$ & $\Gamma$ & $\Delta$ & $\Theta$ & $\Gamma$ & $\Delta$ & $\Theta$ \\
\hline 4.60 & 183.9987 & 644.5488 & 460.5501 & 191.9106 & 635.7698 & 443.8591 & 197.0989 & 630.0409 & 432.9421 \\
\hline 5.00 & 187.7598 & 691.1525 & 503.3926 & 196.2569 & 679.9235 & 483.6665 & 198.8866 & 676.0608 & 477.1742 \\
\hline 5.40 & 191.4837 & 737.2152 & 545.7281 & 200.5359 & 723.5063 & 522.9703 & 203.3135 & 718.9175 & 515.6040 \\
\hline 5.80 & 195.1493 & 782.8152 & 587.6659 & 204.7636 & 766.5681 & 561.8045 & 207.7011 & 761.2520 & 553.5509 \\
\hline 6.20 & 198.7750 & 827.9931 & 629.2181 & 208.9442 & 809.1618 & 600.2176 & 212.0479 & 803.1188 & 591.0709 \\
\hline
\end{tabular}

Table 4 Total costs vs. $v$

\begin{tabular}{|c|c|c|c|c|c|c|c|c|c|}
\hline \multirow{2}{*}{$\begin{array}{l}\alpha \\
v\end{array}$} & \multicolumn{3}{|l|}{0.5} & \multicolumn{3}{|l|}{0.7} & \multicolumn{3}{|l|}{0.9} \\
\hline & $\Gamma$ & $\Delta$ & $\Theta$ & $\Gamma$ & $\Delta$ & $\Theta$ & $\Gamma$ & $\Delta$ & $\Theta$ \\
\hline 0.35 & 98.7895 & 548.2676 & 449.4781 & 95.5000 & 498.4809 & 402.9810 & 92.9471 & 458.5866 & 365.6395 \\
\hline 0.55 & 100.1176 & 554.3015 & 454.1939 & 96.4025 & 508.2268 & 411.8243 & 93.7156 & 472.5236 & 378.8080 \\
\hline 0.75 & 101.9522 & 558.9834 & 457.0311 & 97.8672 & 516.6151 & 418.7479 & 95.1487 & 484.0831 & 388.9344 \\
\hline 0.95 & 104.2273 & 562.3944 & 458.1671 & 99.8645 & 523.7082 & 423.8435 & 97.1571 & 494.2884 & 397.1314 \\
\hline 1.15 & 106.8859 & 565.1780 & 458.2921 & 102.3833 & 529.8369 & 427.4536 & 99.7588 & 502.9528 & 403.1940 \\
\hline
\end{tabular}

Table 5 Total costs vs. $\xi_{2}$

\begin{tabular}{|c|c|c|c|c|c|c|c|c|c|}
\hline \multirow{2}{*}{$\begin{array}{l}\beta \\
\xi_{2}\end{array}$} & \multicolumn{3}{|l|}{0.5} & \multicolumn{3}{|l|}{0.7} & \multicolumn{3}{|l|}{0.9} \\
\hline & $\bar{\Gamma}$ & $\Delta$ & $\Theta$ & $\bar{\Gamma}$ & $\Delta$ & $\Theta$ & $\Gamma$ & $\Delta$ & $\Theta$ \\
\hline 0.79 & 195.2539 & 580.2338 & 384.9799 & 190.4259 & 572.1482 & 381.7223 & 199.3447 & 564.7339 & 365.3892 \\
\hline 0.81 & 195.4810 & 579.6329 & 384.1518 & 191.5068 & 571.3736 & 379.8668 & 201.4988 & 563.8195 & 362.3207 \\
\hline 0.83 & 195.7182 & 579.0377 & 383.3195 & 190.1446 & 572.0249 & 381.8803 & 203.6107 & 562.9214 & 359.3108 \\
\hline 0.85 & 195.9633 & 578.4492 & 382.4859 & 191.1776 & 571.2889 & 380.1113 & 205.6878 & 562.0369 & 356.3491 \\
\hline 0.87 & 196.2156 & 577.8671 & 381.6515 & 192.1779 & 570.5641 & 378.3661 & 207.7247 & 561.1681 & 353.4434 \\
\hline
\end{tabular}

Table 6 Total costs vs. $\xi_{1}$

\begin{tabular}{|c|c|c|c|c|c|c|c|c|c|}
\hline \multirow{2}{*}{$\begin{array}{l}c \\
\xi_{1}\end{array}$} & \multicolumn{3}{|l|}{1} & \multicolumn{3}{|l|}{2} & \multicolumn{3}{|l|}{3} \\
\hline & $\Gamma$ & $\Delta$ & $\Theta$ & $\Gamma$ & $\Delta$ & $\Theta$ & $\Gamma$ & $\Delta$ & $\Theta$ \\
\hline 4.50 & 58.1448 & 198.6960 & 140.5512 & 97.5554 & 397.3824 & 299.8269 & 138.0011 & 596.6616 & 458.6605 \\
\hline 5.00 & 58.1369 & 198.6854 & 140.5485 & 97.5312 & 397.2814 & 299.7502 & 137.9293 & 596.3670 & 458.4377 \\
\hline 5.50 & 58.1297 & 198.6729 & 140.5431 & 97.5028 & 397.1844 & 299.6816 & 137.8431 & 596.0944 & 458.2513 \\
\hline 6.00 & 58.1230 & 198.6588 & 140.5358 & 97.4712 & 397.0918 & 299.6207 & 137.7470 & 595.8445 & 458.0976 \\
\hline 6.50 & 57.2817 & 193.4057 & 136.1240 & 91.7429 & 385.8117 & 294.0688 & 126.7755 & 578.6476 & 451.8720 \\
\hline
\end{tabular}




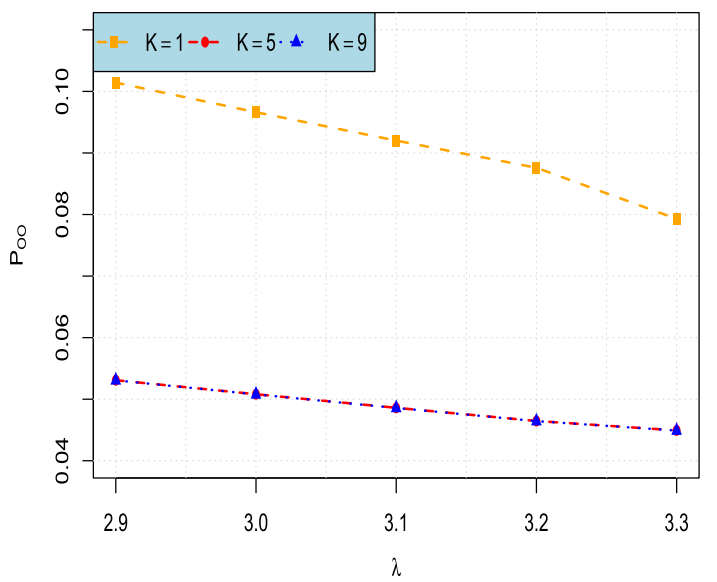

Fig. 2 Impact of $\lambda$ on $P_{0,0}$

$\Delta$ be the total expected revenue per unit time of the system.

$$
\Delta=R \times N_{\mathrm{s}} .
$$

$\Theta$ be the total expected profit per unit time of the system.

$$
\Theta=\Delta-\Gamma \text {. }
$$

\section{Numerical analysis}

In this section, we present numerical examples to analyze the parameter impact on the system performance as well as on total expected cost, total expected revenue, and total expected profit. The characteristics and different costs of the queueing model are obtained using $\mathrm{R}$ program coded by the authors. We assume that the arrival batch size $X$ follows a geometric distribution with parameter $q$, that is $P(X=l)=(1-q)^{l-1} q$, with $0<q<1$, and $l=1,2, \ldots$ Consequently, $B(z)=\frac{q z}{1-(1-q) z}$.

To illustrate the system numerically, the values for default parameters are considered as follows:

First, we consider the following cases:

- Table $1: \lambda=2.9: 0.1: 3.3, K=(1,5,9), c=3, q=0.8, \mu=4, v=3.8, \phi=0.1, \beta=0.8, \alpha=0.8$, $\xi_{1}=0.5$, and $\xi_{2}=0.8$.

- Table $2: \lambda=3, K=3, c=3, q=(0.5,0.7,0.9), \mu=4, v=3.8, \phi=0.07: 0.02: 0.15, \beta=0.8$, $\alpha=0.8, \xi_{1}=0.5$, and $\xi_{2}=0.8$.

- Table 3: $\lambda=3, K=3, c=3, q=0.8, \mu=4.6: 0.4: 6.2, v=3.8, \phi=0.1, \beta=0.8, \alpha=0.8$, $\xi_{1}=0.5$, and $\xi_{2}=(0.8,0.94,1.04)$.

- Table $4: \lambda=3.4, K=3, c=3, q=0.8, \mu=4.0, v=0.35: 0.2: 1.15, \phi=0.1, \beta=0.8$, $\alpha=(0.5,0.7,0.9), \xi_{1}=0.5$, and $\xi_{2}=0.8$.

- Table $5: \lambda=2.9, K=3, c=3, q=0.8, \mu=4.0, v=3.8, \phi=0.1, \beta=(0.5,0.7,0.9), \alpha=0.8$, $\xi_{2}=0.79: 0.02: 0.87$, and $\xi_{1}=0.5$.

- Table 6: $\lambda=3.4, K=3, c=(1,2,3), q=0.8, \mu=4.0, v=3.8, \phi=0.1, \beta=0.8, \alpha=0.8$, $\xi_{1}=4.5: 0.5: 6.5$, and $\xi_{2}=0.8$.

Second, for economic cost results, we consider the following situations: $C_{1}=5, C_{2}=3, C_{3}=4, C_{4}=5$, $C_{5}=5, C_{6}=5, C_{7}=5, C_{8}=4, C_{9}=4, C_{10}=5, C_{11}=4$, and $R=50$. Numerical results are presented in the following tables and figures.

\subsection{Discussion on the results}

- From Table 1 and Figs. 2, 3, we see that for different values of $K$, along the increase of the arrival rate $\lambda$, the probability that the system becomes empty $P_{0,0}$ decreases. Thus, the mean number of customers 


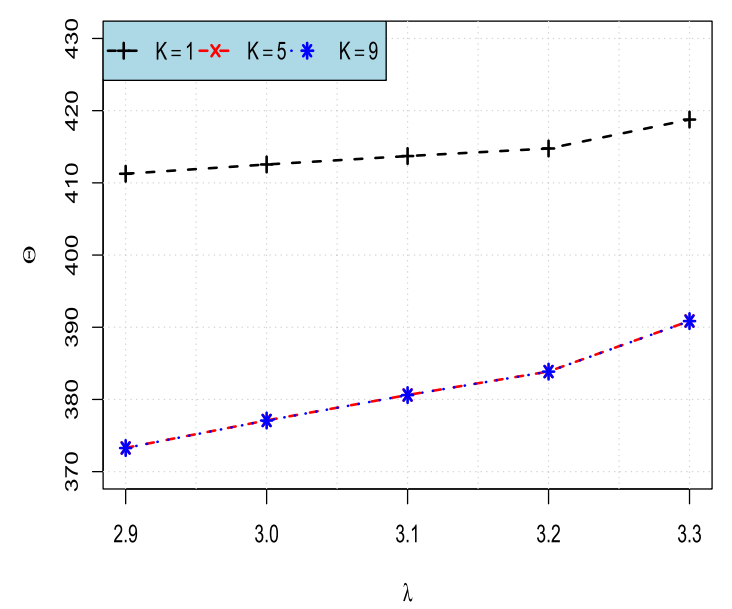

Fig. 3 Impact of $\lambda$ on $\Theta$

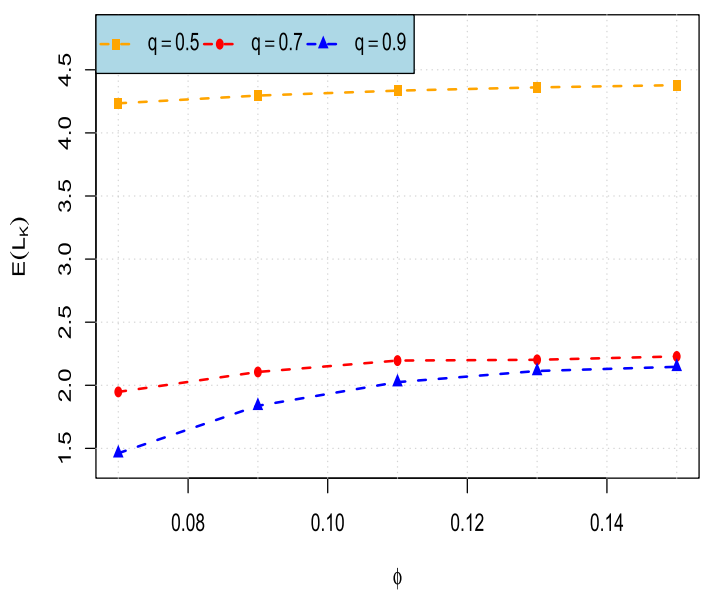

Fig. 4 Impact of $\phi$ on $E\left(L_{\mathrm{K}}\right)$

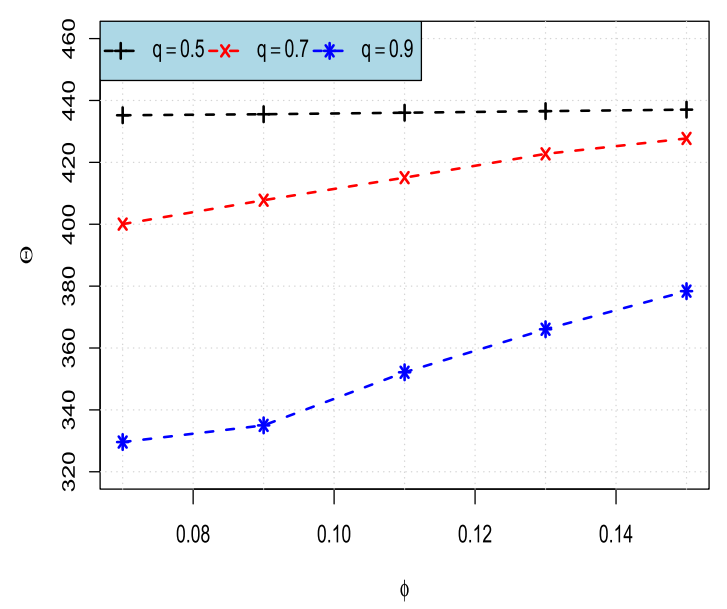

Fig. 5 Impact of $\phi$ on $\Theta$ 


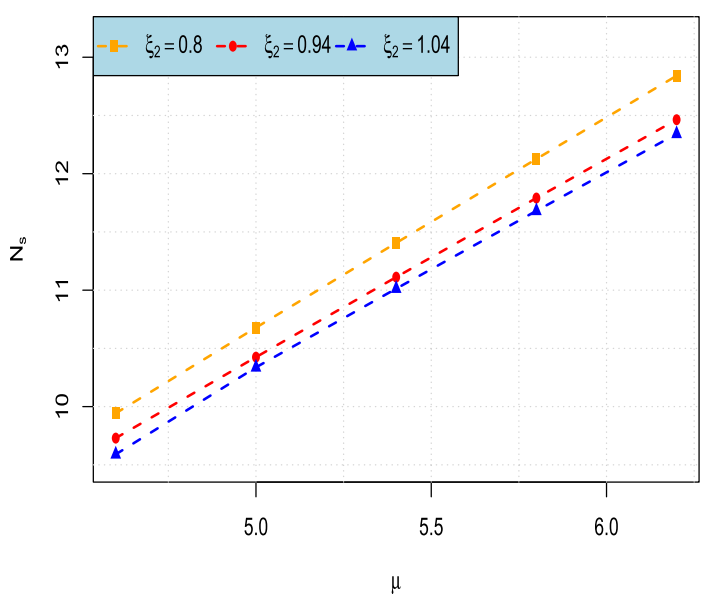

Fig. 6 Impact of $\mu$ on $N_{\mathrm{s}}$

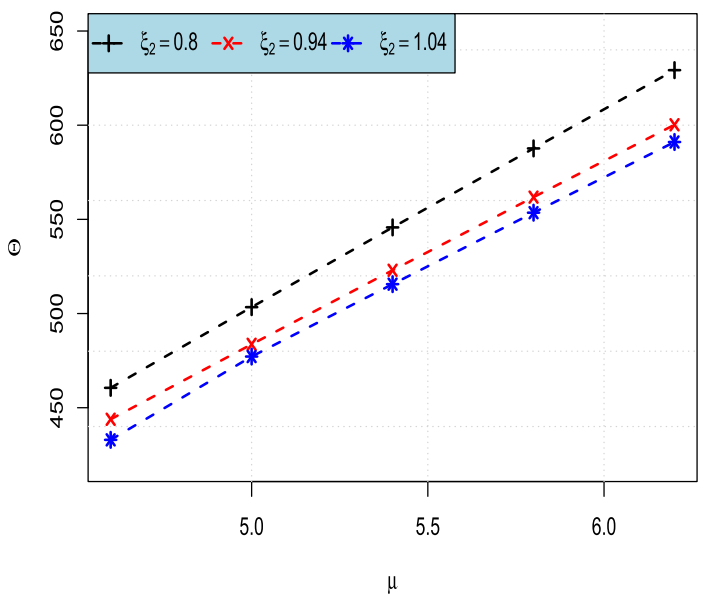

Fig. 7 Impact of $\mu$ on $\Theta$

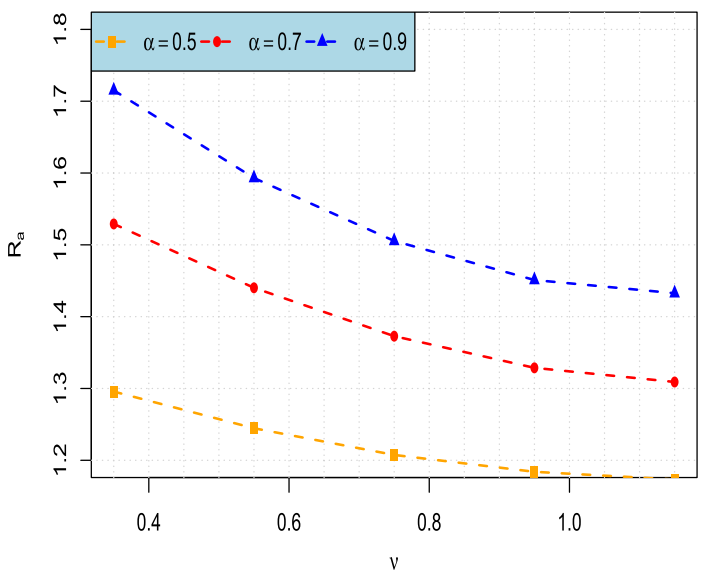

Fig. 8 Impact of $v$ on $R_{\mathrm{a}}$ 


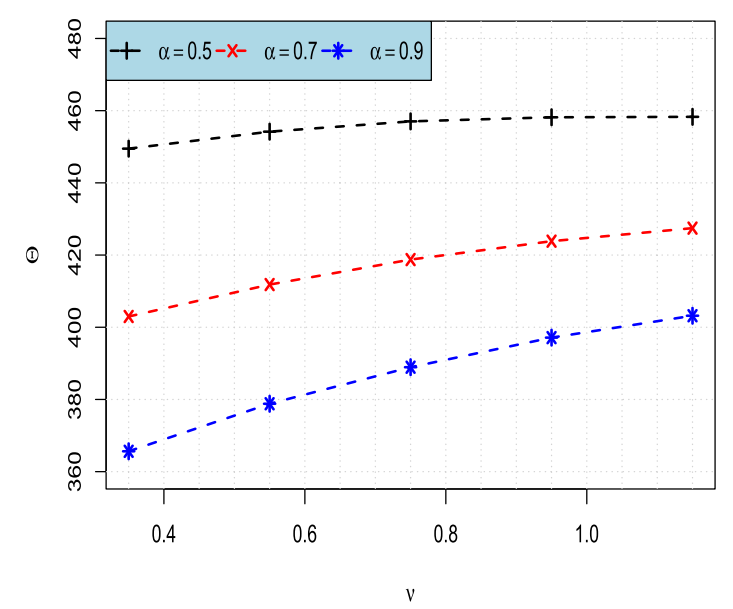

Fig. 9 Impact of $v$ on $\Theta$

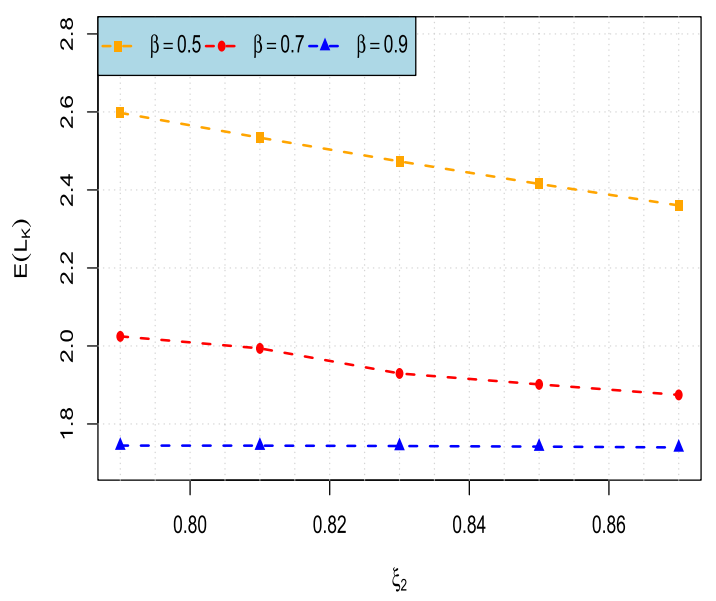

Fig. 10 Impact of $\xi_{2}$ on $E\left(L_{\mathrm{K}}\right)$

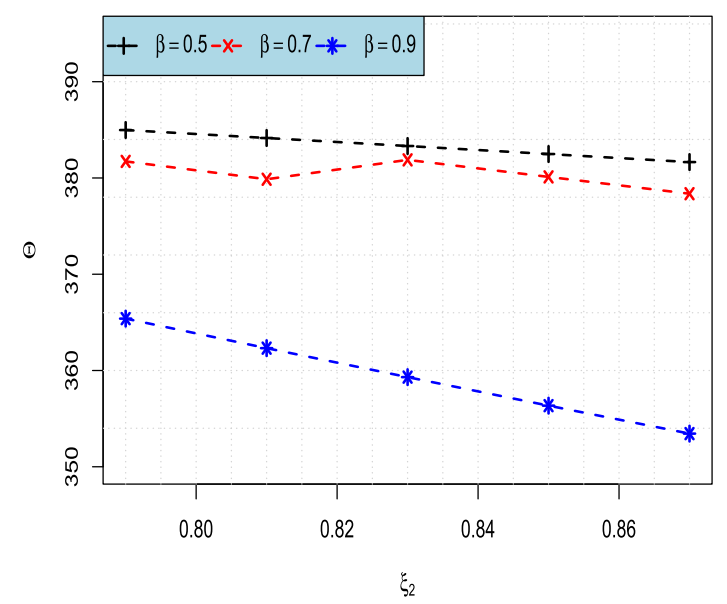

Fig. 11 Impact of $\xi_{2}$ on $\Theta$ 


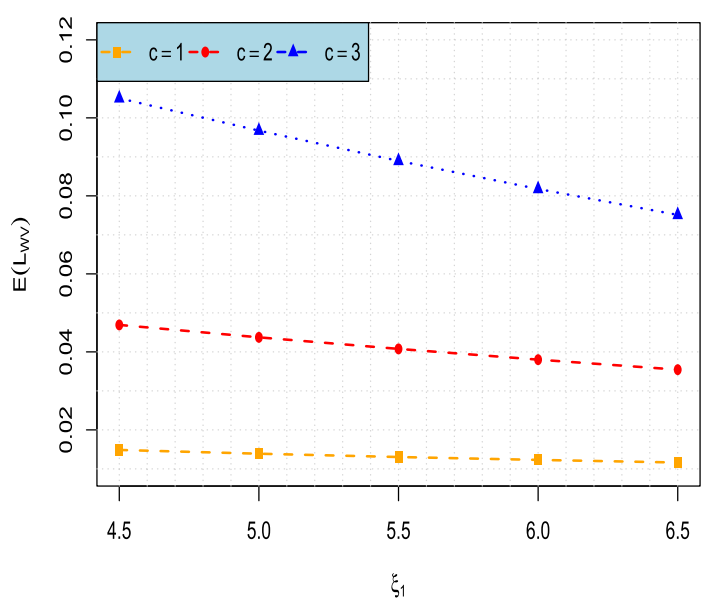

Fig. 12 Impact of $\xi_{1}$ on $E\left(L_{\mathrm{WV}}\right)$

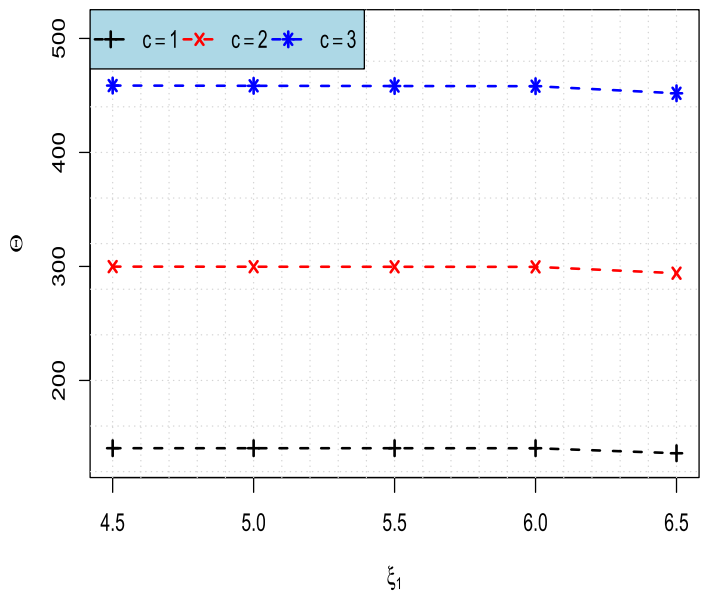

Fig. 13 Impact of $\xi_{1}$ on $\Theta$

served increases. This implies an increase in the total expected profit $\Theta$. Furthermore, it is well observed that the increase of the number of variant vacation has a bad effect on the system.

- The impact of vacation rate $\phi$ is depicted in Table 2 and Figs. 4, 5 for different mean batch sizes $1 / q$. It can be observed that, for fixed $q$, as $\phi$ increases, the mean size of the system when the servers are in normal busy period $E\left(L_{\mathrm{K}}\right)$ increases, as intuitively expected. On the other hand, for fixed $\phi, E\left(L_{\mathrm{K}}\right)$ increases with $1 / q$, as it should be. Thus, it is clearly obvious that the total expected profit $\Theta$ increases with the increasing of $\phi$, while the augmentation of $q$ implies a lost in $\Theta$.

- In Table 3 and Figs. 6, 7, we illustrate the effect of service rate during busy period $\mu$, for various impatience rate during busy period $\xi_{2}$. It is quite clear that with the increase in the service rate $\mu$, the mean number of customers served augments. Thus, the total expected profit $\Theta$ increases. Obviously, the number of customers served decreases when $\xi_{2}$ increases. Thus, we have a significant total expected profit $\Theta$ for large values of $\mu$ and small values of $\xi_{2}$.

- According to the results presented in Table 4 and Figs. 8, 9, we see that the average rate of abandonment $R_{\mathrm{a}}$ decreases with the increases in the service rate during vacation period $v$. This is because the mean number of customers served augments with $v$. Consequently, the average rate of abandonment is reduced. Furthermore, the increase in the probability of non-retention $\alpha$ implies an increase in $R_{\mathrm{a}}$. Finally, it is well observed that the increases in the service rate during vacation period $v$ and in the retention probability $\alpha^{\prime}$ have a nice impact on the total expected profit $\Theta$.

- The impact of the impatience rate during busy period $\xi_{2}$ for different values of non-feedback probabilities $\beta$ is illustrated in Table 5 and Figs. 10, 11. It is clearly shown that, with the increase in impatience rate during normal busy period $\xi_{2}$, the mean size on the system when the servers are in normal busy period $E\left(L_{\mathrm{K}}\right)$ 
decreases, this implies a diminution in the mean number of customers served. Consequently, the total expected profit $\Theta$ decreases. Furthermore, from the above presentations, it is well seen that the feedback probability $\beta^{\prime}$ has a nice effect on the economy of the system.

- Figures 12 and 13 plot the impatience rate during working vacation period $\xi_{1}$ for different values of number of servers $c$. It is well observed that when the impatience rate $\xi_{1}$ is large, the mean size of the system when the servers are on working vacation period decreases. Therefore, the mean number of customers served is reduced. This leads to a decrease in $\Theta$. On the other hand, from Table 6, we observe that when the number of servers becomes large, the total expected profit is significant. This is due to the fact that the mean number of customers served increases with $c$, while the average rate of abandonment decreases with the increasing of the number of the servers.

\section{Conclusions and future scope}

In the present study, we explored reneging behavior in multi-server Bernoulli feedback queueing system with batch arrival, variant of multiple working vacations and retention of the reneged customers. For the analysis purpose, we investigated various system characteristics in terms of steady-state probabilities using the probability generating functions (PGFs). Reneging and retention probabilities incorporated in our model may play an important role in the economy of the concerned system. Numerical experiments performed can be useful and benefic to explore the impacts of system parameters on the performance measures in different contexts. The model developed may provide lucrative perspicacity to the production managers, system engineers, etc. To make the system modeling more closer to the real-world problems, an extension of our results for a nonMarkovian models is a pointer to future research. Moreover, we can extend this study by incorporating the bulk failure.

Open Access This article is distributed under the terms of the Creative Commons Attribution 4.0 International License (http:// creativecommons.org/licenses/by/4.0/), which permits unrestricted use, distribution, and reproduction in any medium, provided you give appropriate credit to the original author(s) and the source, provide a link to the Creative Commons license, and indicate if changes were made.

\section{References}

1. Altman, E.; Yechiali, U.: Infinite-server queues with system's additional tasks and impatient customers. Probab. Eng. Inf. Sci. 22(4), 477-493 (2008)

2. Bouchentouf, A.A.; Yahiaoui, L.: On feedback queueing system with reneging and retention of reneged customers, multiple working vacations and Bernoulli schedule vacation interruption. Arab. J. Math. 6(1), 1-11 (2017)

3. Doshi, B.T.: Single server queues with vacation: a survey. Q. Syst. 1(1), 29-66 (1986)

4. Goswami, V.: Analysis of impatient customers in queues with Bernoulli schedule working vacations and vacation interruption. J. Stoch. 2014, 10 (2014). Article ID 207285

5. Haridass, M.; Arrumuganathan, R.: Analysis of a bulk queue with unreliable server and single vacation. Int. J. Open Probl. Comput. Math. 1(2), 130-148 (2008)

6. Ke, J.C.: Operating characteristic analysis on the $M^{[X]} / G / 1$ system with a variant vacation policy and balking. Appl. Math. Model. 31(7), 1321-1337 (2007)

7. Ke, J.C.; Huang, K.B.; Pearn, W.L.: Randomized policy of a Poisson input queue with J vacations. J. Syst. Sci. Syst. Eng. 19(1), 50-71 (2010)

8. Laxmi, V.P.; Goswami, V.; Jyothsna, K.: Analysis of finite buffer Markovian queue with balking, reneging and working vacations. Int. J. Strategic Decis. Sci. 4(1), 1-24 (2013)

9. Laxmi, V.P.; Jyothsna, K.: Performance analysis of variant working vacation queue with balking and reneging. Int. J. Math. Oper. Res. 6(4), 548-565 (2014)

10. Laxmi, V.P.; Jyothsna, K.: Balking and reneging multiple working vacations queue with heterogeneous servers. J. Math. Model. Algorithm 14(3), 267-285 (2015)

11. Laxmi, V.P.; Rajesh, P.: Analysis of variant working vacations queue with customer impatience. Int. J. Manag. Sci. Eng. Manag. 12(3), 186-195 (2016)

12. Laxmi, V.P.; Rajesh, P.: Performance measures of variant working vacation on batch arrival queue with reneging. Int. J. Math. Arch. 8(8), 85-96 (2017)

13. Madan, K.C.; AI-Rawwash, M.: On the $M^{X} / G / 1$ queue with feedback and optional server vacations based on a single vacation policy. Appl. Math. Comput. 160(3), 909-919 (2005)

14. Perel, N.; Yechiali, U.: Queues with slow servers and impatient customers. Eur. J. Oper. Res. 201(1), 247-258 (2010)

15. Selvaraju, N.; Goswami, C.: Impatient customers in an $M / M / 1$ queue with single and multiple working vacations. Comput. Ind. Eng. 65(2), 207-215 (2013) 
16. Sun, W.; Li, S.: Equilibrium and optimal behavior of customers in Markovian queues with multiple working vacations. TOP 22(2), 694-715 (2014)

17. Takagi, H.: Queueing Analysis, A Foundation of Performance Evaluation, Volume 1: Vacation and Priority Systems, vol. 1. Elsevier, Amsterdam (1991)

18. Tian, N.; Zhang, Z.G.: Vacation Queueing Models: Theory and Applications. International Series in Operations Research and Management Science. Springer, New York (2006)

19. Tian, R.; Hu, L.; Wu, X.: Equilibrium and optimal strategies in $M / M / 1$ queues with working vacations and vacation interruptions. Math. Probl. Eng. 2016, 10 (2016). (Article ID 9746962)

20. Wang, K.H.; Chan, M.C.; Ke, J.C.: Maximum entropy analysis of the $M^{x} / M / 1$ queueing system with multiple vacations and server breakdowns. Comput. Ind. Eng. 52(2), 192-202 (2007)

21. Wang, T.Y.; Ke, J.C.; Chang, F.M.: On the discrete-time $G e o / G / 1$ queue with randomized vacations and at most J vacations. Appl. Math. Model. 35(5), 2297-2308 (2011)

22. Yue, D.; Yue, W.; Xu, G.: Analysis of customers impatience in an $M / M / 1$ queue with working vacations. J. Ind. Manag. Optim. 8(4), 895-908 (2012)

23. Yue, D.; Yue, W.; Saffer, Z.; Chen, X.: Analysis of an $M / M / 1$ queueing system with impatient customers and a variant of multiple vacation policy. J. Ind. Manag. Optim. 10(1), 89-112 (2014)

24. Zhang, Z.G.; Tian, N.: Discrete time Geo/G/1 queue with multiple adaptive vacations. Q Syst. 38(4), 419-429 (2001)

25. Zhang, F.; Wang, J.; Liu, B.: Equilibrium balking strategies in Markovian queues with working vacations. Appl. Math. Model. 37(16-17), 8264-8282 (2013)

Publisher's Note Springer Nature remains neutral with regard to jurisdictional claims in published maps and institutional affiliations. 